\title{
Deciphering porosity clogging at barrier interfaces in deep geological repositories for radioactive waste
}

\author{
Mara I. Lönartz ${ }^{1, \mathbf{i}}$, Jenna Poonoosamy ${ }^{1, \mathbf{i}}$, Yuankai Yang ${ }^{1, \mathbf{i}}$, Naila Ait-Mouheb ${ }^{1, \mathbf{i}}$, Guido Deissmann ${ }^{1, \mathbf{i}}$, \\ and Dirk Bosbach ${ }^{1, \mathbf{i}}$ \\ ${ }^{1}$ Institute of Energy and Climate Research: Nuclear Waste Management and Reactor Safety (IEK-6), \\ Forschungszentrum Jülich GmbH, 52425 Jülich, Germany \\ i All authors are part of the Reactive Transport Modelling group.
}

Correspondence: Mara I. Lönartz (m.loenartz@fz-juelich.de)

Published: 10 November 2021

\begin{abstract}
The disposal of spent nuclear fuels and high-level radioactive wastes in deep geological repositories represents one of the greatest scientific-technical and societal challenges of our times. Most disposal concepts rely on a multibarrier system, consisting of a combination of engineered materials, geotechnical and geological barriers to provide a safe containment of the radioactive waste to protect humans and the environment against dangers arising from ionizing radiation. A reliable safety assessment of a deep geological repository over assessment time scales of several 100000 years requires a profound and comprehensive understanding of the complex coupled physical (thermal, hydraulic, mechanical), chemical and biogeochemical (THM/CB) processes that govern the long-term evolution of the repository system.

As a result of thermal and chemical gradients at the interfaces of different components and materials of the multi-barrier system (e.g. interfaces between metallic waste containers and bentonite backfill or between structural concrete and clay host rock), mineral dissolution and precipitation reactions are promoted; thus the (local) porosity, the volume filled with gas and/or water, can increase or decrease leading to changes in the macroscopic transport properties of the respective media. Although a reduction of the porosity (porosity clogging) appears to be desirable to inhibit radionuclide migration, it can also be detrimental, particularly in the case of gas pressure build-up due to canister corrosion or bacterial activity.

So far, porosity clogging at barrier interfaces and associated consequences on solute or gas transport remain poorly understood; currently used mathematical descriptions of porosity clogging in reactive transport codes usually fail to capture respective experimental observations (Chagneau et al., 2015; Deng et al., 2021). In this context, we are developing a "lab-on-a-chip" set-up, which combines time lapse optical microscopy imaging and in operando Raman spectroscopy (Poonoosamy et al., 2019, 2020) to determine (i) whether complete clogging is possible and permanent, (ii) which parameters control the porosity clogging and (iii) which changes in transport properties of porous media are induced due to porosity clogging. Our approach comprises micronized counterdiffusion experiments with in situ visualization and monitoring of the evolution of mineralogy and microstructure/pore architecture with time. Complementary pore scale modelling will be used to derive key relationships that describe changes in transport properties due to mineral precipitation-induced porosity clogging. This approach will help to improve reactive transport codes and their predictive capabilities thus enhancing confidence and reduce uncertainties in long-term predictions, leading to more realistic descriptions of the evolution of complex repository systems.
\end{abstract}

Kurzfassung. Die Endlagerung abgebrannter Kernbrennstoffe und hochradioaktiver Abfälle in tiefen geologischen Formationen stellt eine der größten wissenschaftlich-technischen und gesellschaftlichen Herausforderungen unserer Zeit dar. Die meisten Entsorgungskonzepte basieren auf einem Multibarrierensystem, das aus einer Kombination von technischen, geotechnischen und geologischen Barrieren besteht, um einen sicheren Einschluss der radioaktiven Abfälle zu gewährleisten, der Mensch und Umwelt vor Gefahren 
durch ionisierende Strahlung schützt. Eine verlässliche Sicherheitsbewertung eines tiefengeologischen Endlagers über Bewertungszeiträume von mehreren 100000 Jahren erfordert ein tiefgreifendes und umfassendes Verständnis der komplexen gekoppelten physikalischen (thermischen, hydraulischen, mechanischen), chemischen und biogeochemischen (THM/CB) Prozesse, die die Langzeitentwicklung des Endlagersystems bestimmen.

Durch thermische und chemische Gradienten an den Grenzflächen zwischen verschiedenen Komponenten und Materialien des Multibarrierensystems (z. B. Grenzflächen zwischen metallischen Abfallbehältern und Bentonitverfüllung oder zwischen Konstruktionsbeton und tonigem Wirtsgestein) werden Auflösungs- und Ausfällungsreaktionen begünstigt; somit kann die (lokale) Porosität, d.h. der mit Gas und/oder Wasser gefüllte Porenraum, zunehmen oder abnehmen, was zu Veränderungen der makroskopischen Transporteigenschaften der jeweiligen Materialien führt. Obwohl eine Verringerung der Porosität (,porosity clogging“) wünschenswert erscheint, um die Radionuklidmigration zu verhindern, kann sie auch von Nachteil sein, insbesondere bei einer Zunahme des Gasdrucks infolge einer Korrosion der Abfallbehälter oder bakterielle Aktivität.

Bis jetzt sind diese Verringerung der Porosität an Barrieregrenzflächen und deren Auswirkungen auf den Transport von Flüssigkeiten oder Gasen nur wenig verstanden, die derzeit verwendeten mathematischen Beschreibungen der Porositätsreduktion in reaktiven Transportcodes werden den entsprechenden experimentellen Beobachtungen in der Regel nicht gerecht (Chagneau et al., 2015; Deng et al., 2021). In diesem Kontext entwickeln wir einen „Labor-auf-dem-Chip“-Aufbau (,lab-on-a-chip“), welcher optische Mikroskopie und konfokale Raman-Spektroskopie kombiniert um zu ermitteln, ob (i) ein vollständiger Verlust der Porosität möglich und von Dauer ist, (ii) welche Parameter die Porositätsverminderung steuern und (iii) welche Veränderungen der Transporteigenschaften poröser Medien durch diese Porositätsveränderungen hervorgerufen werden. Unser Ansatz umfasst mikronisierte Gegendiffusionsexperimente mit In-situ-Visualisierung und Überwachung der zeitlichen Entwicklung von Mineralogie und Mikrostruktur/Porenarchitektur. Eine ergänzende Modellierung auf dem Porenmaßstab wird verwendet, um Stoffgesetze abzuleiten, die Veränderungen der Transporteigenschaften aufgrund einer durch Mineralausfällungen induzierten Porositätsverminderung beschreiben. Dieser Ansatz soll dazu beitragen, reaktive Stofftransportmodelle und ihre Prognosefähigkeiten zu verbessern und Unsicherheiten zu verringern, um eine realistischere Beschreibung der Entwicklung komplexer Endlagersysteme zu erhalten.

Financial support. This research has been supported by the German Federal Ministry of Education and Research (BMBF; grant number 02NUK053A) and the Helmholtz Association (HGF grant SO-093).

\section{References}

Chagneau, A., Claret, F., Enzmann, F., Kersten, M., Heck, S., Madé, B., and Schäfer, T.: Mineral precipitation-induced porosity reduction and its effect on transport parameters in diffusion-controlled porous media, Geochem. T., 16, 1-13, https://doi.org/10.1186/s12932-015-0027-z, 2015.

Deng, H., Tournassat, C., Molins, S., Claret, F., and Steefel, C. I.: A pore-scale investigation of mineral precipitation driven diffusivity change at the column-scale, Water Resour. Res., 57, 1-16, https://doi.org/10.1029/2020WR028483, 2021.
Poonoosamy, J., Westerwalbesloh, C., Deissmann, G., Mahrous, M., Curti, E., Churakov, S. V., Klinkenberg, M., Kohlheyer, D., Lieres, E., Bosbach, D., and Prasianakis, N. I.: A microfluidic experiment and pore scale modelling diagnostics for assessing mineral precipitation and dissolution in confined spaces, Chem. Geol., 528, 119264, https://doi.org/10.1016/j.chemgeo.2019.07.039, 2019.

Poonoosamy, J., Soulaine, C., Burmeister, A., Deissmann, G., Bosbach, D., and Roman, S.: Microfluidic flow-through reactor and 3D Raman imaging for in situ assessment of mineral reactivity in porous and fractured porous media, Lab Chip, 20, 2562-2571, https://doi.org/10.1039/d01c00360c, 2020. 